\title{
Hyperbolicity singularities in Rarefaction Waves
}

\author{
Alexei A. Mailybaev ${ }^{1,3}$ and Dan Marchesin ${ }^{2}$ \\ Received March 7, 2005; Revised March 20, 2006

\begin{abstract}
For mixed-type systems of conservation laws, rarefaction waves may contain states at the boundary of the elliptic region, where two characteristic speeds coincide, and the Lax family of the wave changes. Such contiguous rarefaction waves form a single fan with a continuous profile. Different pairs of families may appear in such rarefactions, giving rise to novel Riemann solution structures. We study the structure of such rarefaction waves near regular and exceptional points of the elliptic boundary and describe their effect on Riemann solutions.
\end{abstract}

KEY WORDS: systems of conservation laws; type change; rarefaction waves; Riemann solutions; classification of singularities; versal deformation.

\section{INTRODUCTION}

Rarefaction waves are the simplest scale invariant solutions for systems of conservation laws. When they involve states where the system is strictly hyperbolic, i.e., all characteristic speeds are real and distinct, these waves are usually extremely stable and well behaved [28]. On the other hand, it is well known that for mixed-type systems of two conservation laws, one cannot construct rarefaction waves containing states in the elliptic region. A number of models studied recently contain elliptic regions, see $[3-5,11,23,25,27]$ and the review in [15]. In these models, rarefaction curves typically contain a point at the boundary of the elliptic region.

In this work, we study rarefaction waves for systems of $m$ conservation laws in the neighborhood of the elliptic boundary defined as the set

\footnotetext{
${ }^{1}$ Institute of Mechanics, Moscow State Lomonosov University, Michurinsky pr. 1, 119192 Moscow, Russia. E-mail: mailybaev@imec.msu.ru.

${ }^{2}$ Instituto Nacional de Matemática Pura e Aplicada - IMPA, Estrada Dona Castorina, 110, 22460-320 Rio de Janeiro RJ, Brazil. E-mail: marchesi@impa.br

${ }^{3}$ To whom correspondence should be addressed.
} 
of states, at which two characteristic speeds coincide. The local structure of rarefaction waves is described near regular and exceptional states of the elliptic boundary. Classification of exceptional points according to the local topological structure of rarefaction curves is given. Explicit formulae providing qualitative and quantitative description of rarefaction curve singularities are derived. These formulae use eigenvectors and associated vectors of coincident characteristic speeds as well as the derivatives up to third order of the flux function at the point of the elliptic boundary. The importance of the third derivative of the flux function is remarkable at exceptional states. As a result, the quadratic approximation is insufficient for local analysis of rarefaction curves near such states. For example, three out of six types of singularities at exceptional points are missing when studying quadratic fluxes $[7,17]$ or quadratic fluxes with small third order terms [8].

Rarefaction waves containing exceptional states play important role in Riemann solutions. We show that, for two types of singularities, they appear in the Riemann solutions for mixed-type problems in a stable way (both structurally and in the Hadamard sense). There are two types of such solutions. Solutions of the first type are known: they are related to the transitional rarefactions for which the family number decreases at the exceptional state $[10,16]$. Solutions of the second type are new: they are related to the rarefactions for which the family number increases at the exceptional state. In the latter case, the local Riemann solution of two conservation laws is a single rarefaction wave. This solution does not change qualitatively under perturbations of the Riemann initial conditions.

For two other singularities, there is an infinite number of Riemann solutions for the same initial data. Despite the fact that these Riemann solutions are structurally stable under perturbations of the Riemann initial conditions, all of them (except for the classical one) are unstable in the Hadamard sense. Therefore they are physically unimportant.

Our method is based on the versal deformation technique $[2,12,13]$ that we use for regularizing the fold structure at the elliptic boundary. The advantage of this method is that both eigenvectors and eigenvalues are evaluated locally in an explicit way. For different constructions for regularizing the rarefaction wave field we refer to $[9,17]$.

The paper is organized as follows. Section 2 contains general information on rarefaction waves. Section 3 describes the versal deformation method for studying the fold structure of rarefaction curves. Sections 4 and 5 study regular and exceptional points of the elliptic boundary. Section 6 is devoted to nonclassical Riemann solutions with singular rarefaction waves. Section 7 gives a numerical example, and the Conclusion 
summarizes the contribution. The formulae required in the versal deformation approach are given in the Appendix. A.

\section{RAREFACTION WAVES IN CONSERVATION LAWS}

Let us consider a system of $m$ conservation laws in one space dimension $x$ :

$$
\frac{\partial U}{\partial t}+\frac{\partial F(U)}{\partial x}=0
$$

where $U(x, t) \in \mathbb{R}^{m}$ is a vector of conserved quantities, and $F \in \mathbb{R}^{m}$ is a flux function dependent smoothly on $U$. For smooth solutions, an alternative form of (2.1) is

$$
\frac{\partial U}{\partial t}+A(U) \frac{\partial U}{\partial x}=0, \quad A(U)=\frac{\partial F}{\partial U},
$$

where $A$ is the $m \times m$ Jacobian matrix of the flux function.

A simple continuous wave solution of (2.2) has the form

$$
U(x, t)=\tilde{U}(\lambda), \quad \lambda=x / t .
$$

This solution represents a rarefaction wave, where each value of $\tilde{U}(\lambda)$ propagates in space $x$ with speed $\lambda$. By substituting (2.3) into equation (2.2), we arrive after elementary manipulations to

$$
A(U) \frac{d U}{\mathrm{~d} \lambda}=\lambda \frac{d U}{\mathrm{~d} \lambda}
$$

if the derivative $\mathrm{d} U / \mathrm{d} \lambda$ exists, or in general to the eigenvalue problem

$$
A(U) r=\lambda r, \quad \mathrm{~d} U \| r,
$$

where $\lambda$ is a real eigenvalue and $r$ is a real eigenvector (here and below we drop tildes over $U$ ). The parallel lines relation $\mathrm{d} U \| r$ means that the eigenvector $r$ of the matrix $A(U)$ is the tangent vector to the rarefaction curve $U(\lambda)$ in state space. In other words, rarefaction curves are introduced locally as integral curves of the line field induced by a real eigenvector of the matrix $A(U)$. The rarefaction wave is defined by a segment of a rarefaction curve under the condition that the corresponding eigenvalue (speed) $\lambda$ increases from the left to the right side of the wave.

The system of conservation laws (2.2) is physically valid in the hyperbolic region of state space $U$, where all the eigenvalues of $A(U)$ are real. If, additionally, all the eigenvalues are distinct, the system is called 
strictly hyperbolic. The values of $U$ such that the matrix $A(U)$ has complex eigenvalues form the elliptic region in state space denoted by $\mathcal{E}$. For generic systems, the boundary between the hyperbolic and elliptic regions consists of hypersurfaces in state space, which are smooth where two eigenvalues coincide forming a $2 \times 2$ Jordan block [2]. At some points, the elliptic boundary may have multiple eigenvalues of other types. But these points form sets of higher codimension, i.e., they appear at singularities (nonsmooth points) of the elliptic boundary.

The existence of a hypersurface with coinciding eigenvalues does not guarantee that there is an elliptic region on one of its sides. In fact, models of Keyfitz-Kranzer-Isaacson-Temple type are physically relevant but maintain hyperbolicity on both sides of the hypersurface [11]. Of course, we do not consider such models in this work.

There are $m$ different rarefaction curves passing through each point in the strict hyperbolicity region, since there are $m$ different eigenvectors corresponding to the eigenvalues (characteristic speeds) $\lambda_{1}(U)<\lambda_{2}(U)<$ $\cdots<\lambda_{m}(U)$ of the matrix $A(U)$. Thus, each eigenvalue $\lambda_{i}(U)$ generates its own family of rarefaction curves. Clearly, the curves inside one family do not intersect and span the whole region of strict hyperbolicity. Curves of different families intersect transversally at each point $U$ with distinct eigenvalues. Points of the rarefaction curves, where the eigenvalue $\lambda$ attains an extremum (maximum or minimum) along the curve, form an inflection locus. The rarefaction wave cannot be extended beyond such states, otherwise the condition of increasing $\lambda$ would be violated.

\section{FOLD STRUCTURE NEAR THE ELLIPTIC BOUNDARY}

Let us consider a point $U_{*}$ at the boundary of the elliptic region, where two eigenvalues couple $\lambda_{i}\left(U_{*}\right)=\lambda_{i+1}\left(U_{*}\right)$ forming a Jordan block (double eigenvalue with a single eigenvector). In the neighborhood of $U_{*}$, the eigenvectors corresponding to $\lambda_{i}(U)$ and $\lambda_{i+1}(U)$ are close to each other and coincide at $U_{*}$. As a result, one expects singular behavior of the rarefaction waves of the $i$ th and $(i+1)$ th families near $U_{*}$. This behavior will be the object of our study.

The matrix $A_{0}=A\left(U_{*}\right)$ has a double eigenvalue $\lambda_{0}=\lambda_{i}\left(U_{*}\right)=\lambda_{i+1}\left(U_{*}\right)$ with a single eigenvector $r_{0}$ and an associated vector $r_{1}$ determined by the Jordan chain equations

$$
A_{0} r_{0}=\lambda_{0} r_{0}, \quad A_{0} r_{1}=\lambda_{0} r_{1}+r_{0} .
$$

The left eigenvector $l_{0}$ and associated vector $l_{1}$ (both $l_{0}$ and $l_{1}$ are row vectors) are given by 


$$
l_{0} A_{0}=\lambda_{0} l_{0}, \quad l_{1} A_{0}=\lambda_{0} l_{1}+l_{0} .
$$

The vectors $r_{0}, r_{1}$ and $l_{0}, l_{1}$ satisfy the relations

$$
l_{0} r_{0}=0, \quad l_{0} r_{1}=l_{1} r_{0} \neq 0
$$

and the orthonormalization conditions

$$
l_{0} r_{1}=1, \quad l_{1} r_{1}=0
$$

determine the unique vectors $l_{0}$ and $l_{1}$ for given $r_{0}$ and $r_{1}$, see e.g. [24].

The point $U_{*}$ belongs to a hypersurface, which is the boundary of the elliptic region, see Figure 1 (the gray area is the elliptic region $\mathcal{E}$ ). When approaching $U_{*}$ from the side of the hyperbolic region, the two eigenvalues $\lambda_{i}(U)$ and $\lambda_{i+1}(U)$ as well as the corresponding eigenvectors approach and merge to $\lambda_{0}$ and $r_{0}$ at $U_{*}$. Hence, the rarefaction curves of the $i$ th and $(i+1)$ th families are both tangent to $r_{0}$ at $U_{*}$.

Eigenvalues and eigenvectors are known to be nonsmooth functions of $U$ at points of multiple eigenvalue, see e.g. [24]. In order to regularize this singularity we consider the real quantities

$$
s=\frac{\lambda_{i}+\lambda_{i+1}}{2}-\lambda_{0}, \quad p=\frac{\left(\lambda_{i+1}-\lambda_{i}\right)^{2}}{4} .
$$

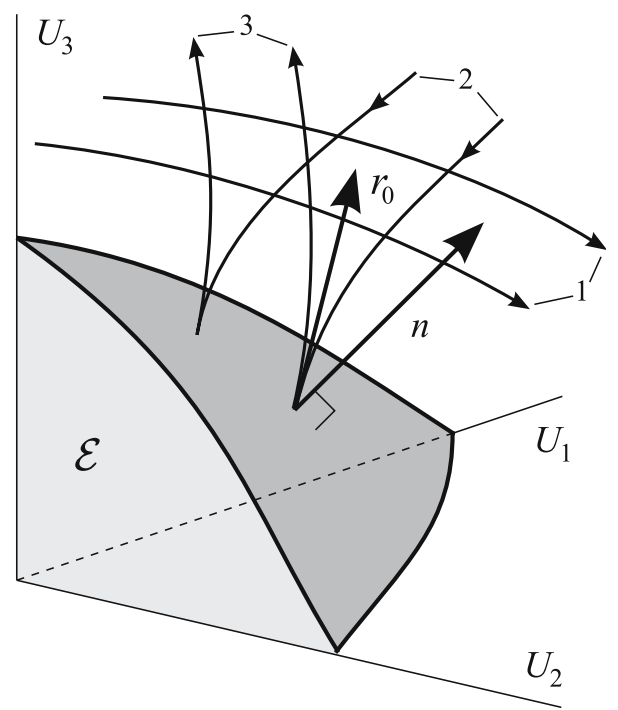

Figure 1. Rarefaction curves for interacting families $(i=2$ and $i+1=3)$ near the elliptic boundary. 
Here $s(U)$ is the average deviation of the interacting eigenvalues $\lambda_{i}$ and $\lambda_{i+1}$ from the coupling value $\lambda_{0}$, and $p(U)$ is the squared half-difference between the eigenvalues. Both $s(U)$ and $p(U)$ are smooth functions of $U$. This follows from a general result in versal deformation theory for nonsymmetric matrices [2,13], which says that the matrix $A(U)$ satisfies the relation

$$
A(U) R(U)=R(U) B(U), \quad B(U)=\left(\begin{array}{cc}
\lambda_{0}+s(U) & 1 \\
p(U) & \lambda_{0}+s(U)
\end{array}\right) .
$$

Here $R(U)=\left[R_{0}(U), R_{1}(U)\right]$ is an $m \times 2$ real matrix that depends smoothly on $U$, and $s(U)$ and $p(U)$ are smooth real scalar functions such that

$$
R_{0}\left(U_{*}\right)=r_{0}, \quad R_{1}\left(U_{*}\right)=r_{1}, \quad s\left(U_{*}\right)=p\left(U_{*}\right)=0 .
$$

At $U_{*}$, the $2 \times 2$ matrix $B\left(U_{*}\right)$ is the Jordan block, and equation (3.6) coincides with (3.1). Derivatives of $s(U), p(U)$, and $R(U)$ at the point $U_{*}$ can be found in terms of the eigenvectors, associated vectors and derivatives of the matrix $A[12,13]$; some of these expressions that are required below are given in the Appendix A.

From (3.6) or, equivalently, from (3.5) we obtain

$$
\lambda_{i}(U)=\lambda_{0}-\sqrt{p(U)}+s(U), \quad \lambda_{i+1}(U)=\lambda_{0}+\sqrt{p(U)}+s(U) .
$$

Using (3.6), it is easy to check that the corresponding eigenvector is

$$
r(U)=R(U)\left(\begin{array}{c}
1 \\
\pm \sqrt{p(U)}
\end{array}\right) \equiv R_{0}(U) \pm \sqrt{p(U)} R_{1}(U),
$$

where the plus and minus signs correspond to $\lambda_{i+1}$ and $\lambda_{i}$, respectively. The eigenvalues (3.8) are real if $p(s) \geq 0$ (hyperbolic region), and complex conjugate if $p(s)<0$ (elliptic region).

In the neighborhood of $U_{*}$, the hyperbolic region is given by the condition $p(s) \geq 0$. Hence, the equation

$$
n \cdot\left(U-U_{*}\right)=0
$$

with $n=\nabla p\left(U_{*}\right)$ gives the tangent hyperplane to the boundary of the elliptic region, where $\nabla=\left(\frac{\partial}{\partial U_{1}}, \ldots, \frac{\partial}{\partial U_{m}}\right)$ is the gradient operator in state space $U=\left(U_{1}, \ldots, U_{m}\right)$, and the dot denotes the inner product in $\mathbb{R}^{m}$. According to (3.10) the vector $n$ is the normal vector to the elliptic boundary at $U_{*}$ directed into the hyperbolic region, see Figure 1. Using the formula (A.5) in the Appendix A, we find

$$
n=\left(l_{0} \frac{\partial A}{\partial U_{1}} r_{0}, \ldots, l_{0} \frac{\partial A}{\partial U_{m}} r_{0}\right) .
$$



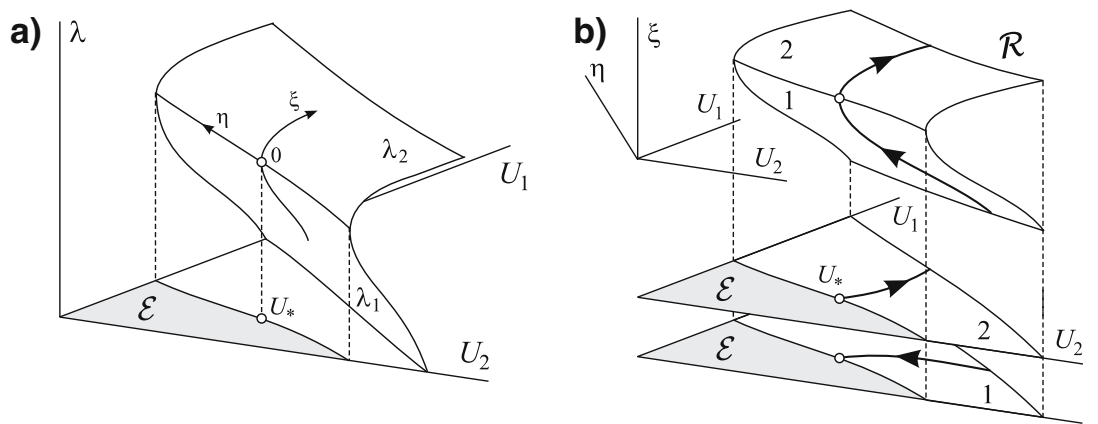

Figure 2. Near the elliptic boundary: (a) new coordinate system $(\xi, \eta)$, (b) lifting state space into $(\xi, \eta, U)$ space.

In the case of two conservation laws, $U=\left(U_{1}, U_{2}\right)$, the eigenvalues $\lambda_{1}$ and $\lambda_{2}$ are represented by two sheets in the $\left(U_{1}, U_{2}, \lambda\right)$ space. These two sheets are connected along the points with coinciding eigenvalues $\lambda_{1}=$ $\lambda_{2}$, see Figure 2(a) (complex eigenvalues in the elliptic region are disregarded). Using expressions (3.5), where $p(U)$ and $s(U)$ are smooth functions, one can show that the $\lambda_{1}$ and $\lambda_{2}$ eigenvalue sheets put together form a smooth surface in the $\left(U_{1}, U_{2}, \lambda\right)$ space, as follows. We introduce a coordinate system $(\xi, \eta)$ on this surface

$$
\xi^{2}=p(U), \quad \eta=s(U) .
$$

Clearly, the real variable $\xi$ is defined only in the hyperbolic region $p(U) \geq 0$. The eigenvalues (3.8) and eigenvectors (3.9) in the new coordinate system become

$$
\lambda=\lambda_{0}+\xi+\eta, \quad r=R(U)\left(\begin{array}{l}
1 \\
\xi
\end{array}\right) \equiv R_{0}(U)+\xi R_{1}(U),
$$

where $\xi<0$ and $\xi>0$ correspond to the lower $\left(\lambda_{1}\right)$ and upper $\left(\lambda_{2}\right)$ eigenvalue sheets, respectively. The coinciding eigenvalues appear exactly at the $\eta$ axis, and they are equal to $\lambda=\lambda_{0}+\eta$. In the general case $m>2$, the $m$-dimensional surfaces corresponding to $\lambda_{i}(U)$ and $\lambda_{i+1}(U)$ in the $(U, \lambda)$ space join at the coupling points $\lambda_{i}=\lambda_{i+1}$. Together they form one smooth $m$-dimensional surface, which is parametrized by taking $\xi, \eta$, and $m-2$ components of the state vector $U$.

For us it is convenient to work in the $(m+2)$-dimensional space $(\xi, \eta, U)$, which contains the smooth $m$-dimensional surface $\mathcal{R}$ given by conditions (3.12). The coordinates in this space provide a smooth parametrization of the eigenvalue and eigenvector (3.13). The line field in state 
space $U$ given by the relation $d U \| r$ induces the line field in the $(\xi, \eta, U)$ space. Using (3.12) and assuming that $\xi \neq 0$, we obtain

$$
(\mathrm{d} \xi, \mathrm{d} \eta, \mathrm{d} U) \|\left(\frac{\nabla p \cdot \mathrm{d} U}{2 \xi}, \nabla s \cdot \mathrm{d} U, \mathrm{~d} U\right) .
$$

Multiplying the right-hand side of (3.14) by the scalar $2 \xi$ and using the relation $d U \| r$, we get the equivalent form for the line field as

$$
(\mathrm{d} \xi, \mathrm{d} \eta, \mathrm{d} U) \|(\nabla p \cdot r, 2 \xi \nabla s \cdot r, 2 \xi r) .
$$

Now we introduce the vector field

$$
\left(\begin{array}{c}
\dot{\xi} \\
\dot{\eta} \\
\dot{U}
\end{array}\right)=\left(\begin{array}{c}
\nabla p \cdot r \\
2 \xi \nabla s \cdot r \\
2 \xi r
\end{array}\right)
$$

associated with the line field (3.15), where the dot over a symbol denotes differentiation with respect to the parametrization variable $\tau$. Notice that this vector field depends smoothly on $\xi$ and $U$, and does not depend on $\eta$.

The integral curves of the line field (3.15) coincide with the integral curves of the vector field (3.16). Projecting these integral curves (which lie in the surface $\mathcal{R}$ given by (3.12)) onto the $U$ space yields the rarefaction curves. Thus, the main idea of our construction is lifting two "copies" of hyperbolic parts of state space $U$ in the $(\xi, \eta, U)$ space so that they glue forming a smooth surface, see Figure 2(b). The lower and upper parts of this surface correspond to the $i$ th and $(i+1)$ th families so that the corresponding rarefaction curves become the integral curves for the explicit smooth vector field (3.16).

\section{REGULAR POINTS OF THE ELLIPTIC BOUNDARY}

Let $U_{*}$ be a point at the boundary of the elliptic region. As $p\left(U_{*}\right)=$ $s\left(U_{*}\right)=0$, the point $U_{*}$ lifted into the $(\xi, \eta, U)$ space becomes $\left(0,0, U_{*}\right)$. The vector field $(3.16)$ at $\left(0,0, U_{*}\right)$ takes the form $\left(n \cdot r_{0}, 0,0\right)$.

In this section, we consider the nonsingular case $n \cdot r_{0} \neq 0$ when the (lifted) vector field has no singularity. Using expression (3.11) and the relation $A=\nabla F$, we write the regularity condition $n \cdot r_{0} \neq 0$ as

$$
n \cdot r_{0}=l_{0} d^{2} F\left(r_{0}, r_{0}\right) \neq 0 .
$$


Here $d^{2} F(a, b)$ denotes the second derivative of the flux function at $U_{*}$ :

$$
d^{2} F(a, b)=\left.\sum_{i, j=1}^{m} \frac{\partial^{2} F}{\partial U_{i} \partial U_{j}}\right|_{U=U_{*}} a_{i} b_{j}, \quad a, b \in \mathbb{R}^{m} ;
$$

below we will also use a similar notation for the first and third derivatives $d F(a), d^{3} F(a, b, c)$ taken at $U_{*}$. Recall that $n$ is the normal vector to the elliptic boundary at $U_{*}$. Thus, the condition $n \cdot r_{0} \neq 0$ implies that the eigenvector $r_{0}$ is not tangent to the elliptic boundary.

Using expressions (3.7) and (3.13), we expand the right-hand side of equation (3.16) into Taylor series at $\left(0,0, U_{*}\right)$ retaining terms up to fist order:

$$
\left(\begin{array}{c}
\dot{\xi} \\
\dot{\eta} \\
\dot{U}
\end{array}\right)=\left(\begin{array}{c}
n \cdot r_{0}+\xi n \cdot r_{1}+h \cdot\left(U-U_{*}\right) \\
2 \xi q \cdot r_{0} \\
2 \xi r_{0}
\end{array}\right),
$$

where the vectors $h$ and $q$ are defined as

$$
\begin{aligned}
& h \cdot a=d^{2} p\left(a, r_{0}\right)+n \cdot d R_{0}(a), \\
& q \cdot a=\nabla s\left(U_{*}\right) \cdot a=\frac{1}{2} l_{0} d^{2} F\left(a, r_{1}\right)+\frac{1}{2} l_{1} d^{2} F\left(a, r_{0}\right), \quad a \in \mathbb{R}^{m} .
\end{aligned}
$$

Here we used formula (A.6) in the Appendix A for derivatives of the function $s(U)$. The first derivatives of $R_{0}(U)$ and second derivatives of $p(U)$ at $U_{*}$ are given by the formulae (A.9) and (A.13). Solving (4.3) yields

$$
\begin{aligned}
\xi & =\left(n \cdot r_{0}\right) \tau+o(\tau), \\
\eta & =\eta_{0}+\left(q \cdot r_{0}\right)\left(n \cdot r_{0}\right) \tau^{2}+o\left(\tau^{2}\right), \\
U & =U_{0}+r_{0}\left(n \cdot r_{0}\right) \tau^{2}+o\left(\tau^{2}\right),
\end{aligned}
$$

where we assume $\xi_{0}=\xi(0)=0$, which can always be achieved by a shift in the parameter $\tau$. The integral curves (4.5) have orthogonal intersection at $\tau=0$ with the $\xi=0$ hyperplane, see Figure 3(a).

There is a single integral curve passing through $\left(0,0, U_{*}\right)$, for which $\eta_{0}=0$ and $U_{0}=U_{*}$. When projecting into state space $U$, the lower part of this integral curve $(\xi<0)$ becomes a rarefaction curve of the $i$ th family, while the upper part $(\xi>0)$ becomes a rarefaction curve of the $(i+1)$ th family. Using the expression (3.13) for the speed of the rarefaction wave $\lambda$, we find from (4.5) that

$$
U(\lambda)=U_{*}+\frac{\left(\lambda-\lambda_{0}\right)^{2}}{n \cdot r_{0}} r_{0}+o\left(\left|\lambda-\lambda_{0}\right|^{2}\right) .
$$



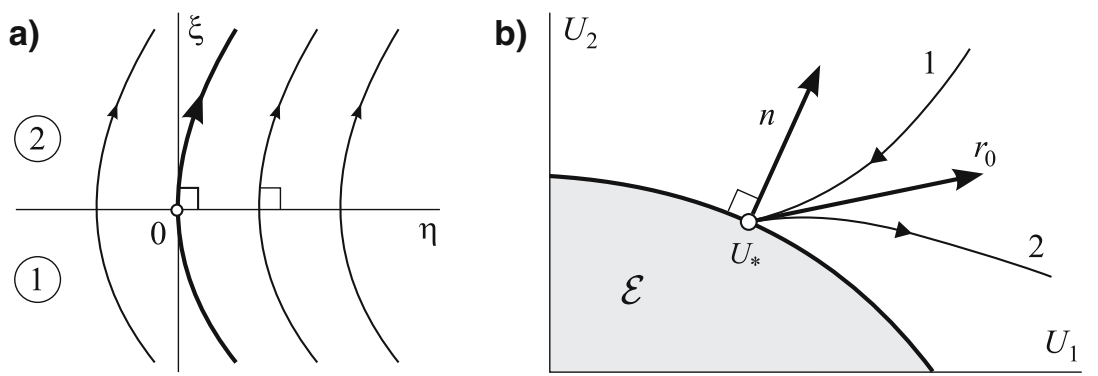

Figure 3. Rarefaction waves near a regular point of the elliptic boundary for two conservation laws: (a) in $(\xi, \eta)$ coordinates, (b) in state space.

When $\lambda<\lambda_{0}$, expression (4.6) provides an approximation for the rarefaction wave of the $i$ th family: as $\lambda$ tends to $\lambda_{0}$, the state $U$ approaches the elliptic boundary along the eigenvector $r_{0}$. When $\lambda>\lambda_{0}$, expression (4.6) describes the rarefaction wave of the $(i+1)$ th family. In this case, the state $U$ moves away from the elliptic boundary along the eigenvector $r_{0}$ when $\lambda$ increases from $\lambda_{0}$. Figure 3(b) describes this behavior for the case $m=2$, when $i=1$ and $i+1=2$. Equation (4.6) is to be contrasted with the classical expression for $U$ on the $i$ th rarefaction wave near the state $U_{0}=U\left(\lambda_{0}\right)$ outside the elliptic region, away from the inflection locus

$$
U(\lambda)=U_{0}+\frac{\lambda-\lambda_{0}}{\nabla \lambda \cdot r_{i}} r_{i}+O\left(\left|\lambda-\lambda_{0}\right|^{2}\right)
$$

Using expression (3.13) and equations (4.3), we find the derivative of the speed $\lambda$ along the integral curve as $\dot{\lambda}=\dot{\xi}+\dot{\eta} \approx n \cdot r_{0}$. This derivative is nonzero and does not change sign near the point $U_{*}$. Hence, there can be no inflection points (i.e., where $\lambda$ has an extremum) in the neighborhood of regular points of the elliptic boundary.

\section{EXCEPTIONAL POINTS AT THE ELLIPTIC BOUNDARY}

Let us consider a point $U_{*}$ at the elliptic boundary and assume that $n \cdot r_{0}=0$, i.e., the eigenvector $r_{0}$ is tangent to the elliptic boundary violating the regularity inequality (4.1). We will call such points exceptional. The set of exceptional points on the elliptic boundary is given by the condition $\nabla p(U) \cdot r(U)=0$, where $\nabla p$ is a normal vector to the boundary in state space and $r$ is an eigenvector with the double eigenvalue $\lambda_{0}$. Using expression (3.13) and the equality $\xi=0$ at the elliptic boundary, we write this condition in the form $\nabla p(U) \cdot R_{0}(U)=0$. Linearizing near the exceptional point $U_{*}$, we obtain 


$$
\nabla p(U) \cdot R_{0}(U) \approx h \cdot\left(U-U_{*}\right)=0,
$$

where the vector $h$ evaluated at $U_{*}$ is introduced in (4.4); in the derivation we used the relations $n=\nabla p\left(U_{*}\right)$ and $R_{0}\left(U_{*}\right)=r_{0}$. The linear equation (5.1) together with the approximation of the elliptic boundary (3.10) provide the tangent plane to the set of exceptional points. In the generic case, when the vectors $\nabla p$ and $h$ are linearly independent, the set of exceptional points is a smooth surface of codimension 2 in state space $U$. In the case of two conservation laws $\left(U \in \mathbb{R}^{2}\right)$, exceptional points are isolated points on the elliptic boundary curve. In the case $U \in \mathbb{R}^{3}$, exceptional points form smooth curves on the elliptic boundary surface.

\subsection{Inflection Locus}

The inflection locus is given by the points in state space where the speed $\lambda$ attains a maximum or a minimum along the rarefaction curve. In the space $(\xi, \eta, U)$ this condition becomes

$$
\dot{\lambda}=\dot{\xi}+\dot{\eta}=\nabla p \cdot r+2 \xi \nabla s \cdot r=0,
$$

where expressions (3.13) and (3.16) were used. Equation (5.2) defines a smooth hypersurface in the $(\xi, \eta, U)$ space through the point $\left(0,0, U_{*}\right)$ with tangent plane given by the linearized equation

$$
\nabla p \cdot r+2 \xi \nabla s \cdot r \approx\left(n \cdot r_{1}+2 q \cdot r_{0}\right) \xi+h \cdot\left(U-U_{*}\right)=0 .
$$

Therefore, the inflection locus lifted in the $(\xi, \eta, U)$ space is a smooth codimension 1 hypersurface. For two conservation laws, it is a smooth curve in the $(\xi, \eta)$ plane defined by equation $(5.2)$, where the vector $U$ is given implicitly by equations (3.12).

Using (3.13), we write equation (5.2) in the form

$$
\xi=-\frac{2 \xi^{2} \nabla s \cdot R_{1}+\nabla p \cdot R_{0}}{2 \nabla s \cdot R_{0}+\nabla p \cdot R_{1}} .
$$

Now, by squaring both sides in (5.4) and using the relation $\xi^{2}=p(U)$ from (3.12), we find the equation for the inflection locus in state space as

$$
p=\left(\frac{2 p \nabla s \cdot R_{1}+\nabla p \cdot R_{0}}{2 \nabla s \cdot R_{0}+\nabla p \cdot R_{1}}\right)^{2},
$$

where $p, s, R_{0}$, and $R_{1}$ are smooth functions of $U$. At the exceptional point $U=U_{*}$, we have $2 p \nabla s \cdot R_{1}+\nabla p \cdot R_{0}=n \cdot r_{0}=0$ and $2 \nabla s \cdot R_{0}+\nabla p$. $R_{1}=2 q \cdot r_{0}+n \cdot r_{1}$. Hence, under the nondegeneracy condition $2 q \cdot r_{0}+n$. $r_{1} \neq 0$, the inflection locus is a smooth hypersurface in state space defined 
by equation (5.5); its tangent plane at $U_{*}$ is given by $n \cdot\left(U-U_{*}\right)=0$. Recall that $n$ is the normal vector to the elliptic boundary. Therefore, the inflection locus is a smooth codimension 1 hypersurface in state space, which is tangent to the elliptic boundary at exceptional points. In particular, it is a smooth curve in the case of two conservation laws.

\subsection{Linearization of the Vector Field}

One can see that, since $n \cdot r_{0}$ stands in the denominator of (4.6), the behavior of the rarefaction waves should change near exceptional points. Indeed, $\left(0,0, U_{*}\right)$ is an equilibrium of the vector field (3.16). Under the condition $n \cdot r_{0}=0$, the linearized vector field (4.3) near $\left(0,0, U_{*}\right)$ takes the form

$$
\left(\begin{array}{c}
\dot{\xi} \\
\dot{\eta} \\
\dot{U}
\end{array}\right)=\left(\begin{array}{ccc}
n \cdot r_{1} & 0 & h^{T} \\
2 q \cdot r_{0} & 0 & 0 \\
2 r_{0} & 0 & 0
\end{array}\right)\left(\begin{array}{c}
\xi \\
\eta \\
U-U_{*}
\end{array}\right) .
$$

The eigenvalues $\rho$ of the $(m+2) \times(m+2)$ matrix in the right-hand side of (5.6) are found from the characteristic equation

$$
\rho^{m}\left(\rho^{2}-\left(n \cdot r_{1}\right) \rho-2 h \cdot r_{0}\right)=0 .
$$

There is a zero eigenvalue $\rho=0$ of multiplicity $m$. The corresponding eigenspace has dimension $m$ and consists of the eigenvectors

$$
w_{0}=\left(\begin{array}{l}
0 \\
c \\
\hat{r}
\end{array}\right),
$$

where $c \in \mathbb{R}$ and $\hat{r}$ is any vector satisfying the orthogonality condition $h$. $\hat{r}=0$. The two remaining eigenvalues are

$$
\rho_{ \pm}=\frac{1}{2}\left(n \cdot r_{1} \pm \sqrt{\left(n \cdot r_{1}\right)^{2}+8 h \cdot r_{0}}\right)
$$

with the corresponding eigenvectors

$$
w_{ \pm}=\left(\begin{array}{c}
\rho_{ \pm} \\
2 q \cdot r_{0} \\
2 r_{0}
\end{array}\right) .
$$



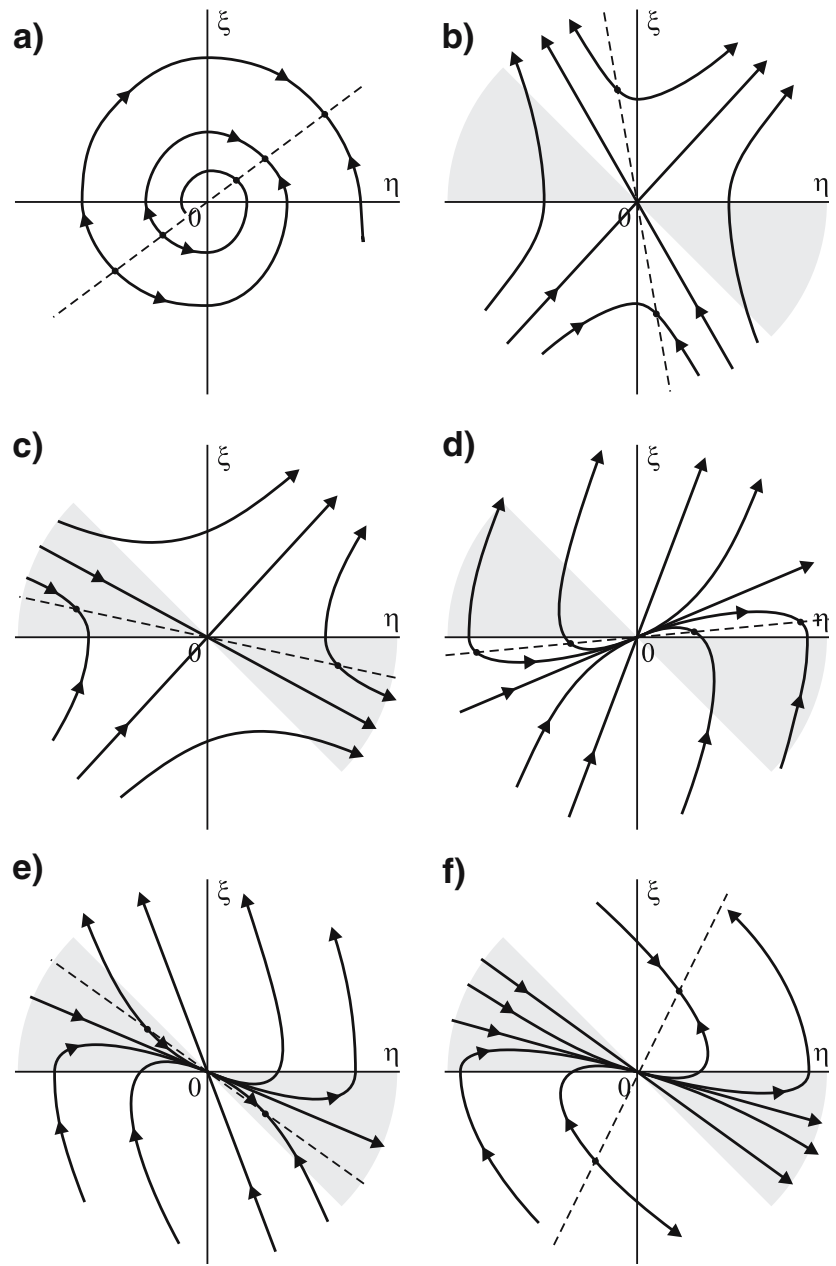

Figure 4. Generic structures of integral curves near exceptional points in the $(\xi, \eta)$ plane for the linearized vector field. Arrows show the direction of increasing speed $\lambda$; the dashed line is the inflection locus; $\eta$-axis is the fold line.

\subsection{Singularities of Integral Curves}

Let us study the structure of integral curves in the nondegenerate case when $\rho_{ \pm} \neq 0, \rho_{-}<\rho_{+}, q \cdot r_{0} \neq 0$, and $\rho_{ \pm} \neq-2 q \cdot r_{0}$. Violation of these conditions leads to higher codimension singularities, which are out of the scope of our paper. We assume that $q \cdot r_{0}>0$, which can be achieved by choosing appropriately the direction of the eigenvector $r_{0}$. 
Table I. Singularities in Figure 4 (assumptions: $q \cdot r_{0}>0$ and $\rho_{-}<\rho_{+}$). The fourth column gives the numbers of exceptional integral curves corresponding to the types $(i,+),(i,-)$, $(i+1,+),(i+1,-)$ (here $i$ or $i+1$ is the family number, and + or - determines whether the characteristic speed increases or decreases away from the singularity).

\begin{tabular}{lllll}
\hline Case & Equilibrium & $\begin{array}{l}\text { Additional } \\
\text { conditions }\end{array}$ & $\begin{array}{c}\text { Exceptional } \\
\text { integral curves }\end{array}$ & $\begin{array}{c}\text { Simplest } \\
\text { flux }\end{array}$ \\
\hline$a$ & Focus & & $0,0,0,0$ & Cubic \\
$b$ & Saddle & $\rho_{-}<-2 q \cdot r_{0}$ & $0,2,2,0$ & Quadratic \\
$c$ & Saddle & $\rho_{-}>-2 q \cdot r_{0}$ & $1,1,1,1$ & Quadratic \\
$d$ & Node & $\begin{array}{c}\rho_{-}>0 \\
\left(\text { or } \rho_{+}<-2 q \cdot r_{0}\right)\end{array}$ & $0, \infty, \infty, 0$ & Quadratic \\
$e$ & Node & $\rho_{-}<-2 q \cdot r_{0}<\rho_{+}<0$, & $\infty, 1,1, \infty$ & Cubic \\
$f$ & Node & $-2 q \cdot r_{0}<\rho_{-}<\rho_{+}<0$ & $\infty, 0,0, \infty$ & Cubic \\
\hline
\end{tabular}

The center manifold of the equilibrium $\left(0,0, U_{*}\right)$ in $(\xi, \eta, U)$ space is the set of equilibria (the codimension-2 surface of exceptional points). According to the reduction principle [1], the local dynamics is topologically equivalent to the dynamics induced by the linearized field on the stable and unstable manifolds, together with the nonlinear dynamics on the center manifold. The latter dynamics is trivial since this center manifold consists of equilibria. So we conclude that the local dynamics is topologically equivalent to the vector field given by the linearized system, which is considered below.

The two eigenvectors $w_{ \pm}$define a 2-dimensional invariant plane of the linearized vector field (5.6) at the exceptional point $U_{*}$. Invariant planes with the same integral curve structure are obtained for all nearby exceptional points by shifting the plane through $U_{*}$ in the direction of the eigenvectors $w_{0}$. Thus, the $(\xi, \eta)$ plane coordinates facilitate the study of the integral curves structure in the invariant plane; the coordinates $U$ are determined uniquely by $(\xi, \eta)$ and can be suppressed. Figure 4 shows all six generic structures of integral curves near the exceptional points, corresponding to the cases described in Table I. They are distinguished by several features. One is the equilibrium type: focus (complex $\rho_{ \pm}$), saddle (real $\rho_{ \pm}, \rho_{-} \rho_{+}<0$ ), or node (real $\rho_{ \pm}, \rho_{-} \rho_{+}>0$ ). Another criterion is the number and the type of the integral curves starting or finishing at the equilibrium. These curves exist if $\rho_{ \pm}$are real, and they are tangent to the eigenvectors $w_{ \pm}$.

Recall that the lower $(\xi<0)$ and the upper $(\xi>0)$ parts of the $(\xi, \eta)$ plane correspond to the $i$ th and $(i+1)$ th characteristic families of rarefaction waves, respectively. Points on the line $\xi=0$ for small nonzero $\eta$ correspond to regular points of the elliptic boundary. In the $(\xi, \eta)$ plane, the 
integral curves are orthogonal to the $\eta$ axis at regular boundary points, a fact that can be shown by taking $\xi=0$ in (5.6).

Since the speed of the rarefaction wave is $\lambda=\lambda_{0}+\xi+\eta$, the direction along the integral curve where $\lambda$ increases is easily determined by the sign of the variation $d \lambda=d \xi+d \eta$. Increasing $\lambda$ is shown by arrows in Figure 4. We distinguish the kind of exceptional curve near $\left(0,0, U_{*}\right)$ according to the family and the increasing speed direction. The family number of the curve near the origin is $i$ for $\xi<0$ and $i+1$ for $\xi>0$. We denote by "+" the situation when the speed $\lambda$ increases as we move along the curve away from the origin; otherwise, we write "-". The number of each kind of integral curve is given in Table I for each singularity type. Near the origin, the $(i,+)$ and $(i+1,-)$ exceptional curves lie in the gray sectors in Figure 4, which are given by the conditions $-1<\xi / \eta<0$. Remark that, in case $a$, there is an infinite number of spiral integral curves starting at the equilibrium, but they have neither definite family nor speed direction near the origin. The inflection locus (5.2) is shown in Figure 4 by dashed lines.

Finally, we remark that in the case $\rho_{-} \rho_{+}>0\left(\operatorname{Re} \rho_{-}=\operatorname{Re} \rho_{+}\right)$, there is a 2-dimensional smooth invariant manifold through each exceptional point tangent to the eigenvectors $w_{ \pm}$: it is the stable or unstable manifold depending on the sign of $\operatorname{Re} \rho_{ \pm}$. These manifolds give locally a foliation of $(\xi, \eta, U)$ space. If $\rho_{-} \rho_{+}<0\left(\operatorname{Re} \rho_{-} \neq \operatorname{Re} \rho_{+}\right)$, the smooth invariant manifold tangent to $w_{ \pm}$exists in case of two conservation laws: it is the two-dimensional surface $\mathcal{R}$ (see Section 3). For $m>2$, there are onedimensional stable and unstable manifolds tangent to the vectors $w_{ \pm}$. The existence of a smooth two-dimensional invariant manifold is a nontrivial question, since the reduction principle provides only the topological (homeomorphic) equivalence.

\subsection{Two Conservation Laws}

For two conservation laws, there are two characteristic speeds $\lambda_{1}$ and $\lambda_{2}$, which coincide at the boundary of the elliptic region. Generically, the elliptic boundary is a smooth curve in state space $U \in \mathbb{R}^{2}$. The eigenvector $r_{0}$ of the double eigenvalue $\lambda_{1}=\lambda_{2}$ is tangent to this curve at the exceptional points. Let $U_{*}$ be an exceptional point. For two dimensions, the $2 \times 2$ matrix $G$ introduced in the Appendix A becomes

$$
G=A_{0}-\lambda_{0} I+r_{1} l_{1}=r_{0} l_{0}+r_{1} l_{1} .
$$

In particular, one can easily check by using (3.3) and (3.4) that $G^{-1}=G$. Then, the eigenvalues (5.9) can be determined by means of expressions 
(4.4), (5.11), the condition $n \cdot r_{0}=0$ at $U_{*}$, formulae (A.5), (A.6), (A.9), (A.12) in the Appendix A, and the relation $A=\nabla F$. Elementary manipulations enable these eigenvalues to be expressed as

$$
\begin{aligned}
& \rho_{ \pm}=\frac{1}{2}\left(l_{0} d^{2} F\left(r_{0}, r_{1}\right) \pm \sqrt{D}\right), \\
& D=\left(3 l_{0} d^{2} F\left(r_{0}, r_{1}\right)-2 l_{1} d^{2} F\left(r_{0}, r_{0}\right)\right)^{2}+8 l_{0} d^{3} F\left(r_{0}, r_{0}, r_{0}\right) .
\end{aligned}
$$

One can see that expressions (5.12) require second derivatives of the flux function $F$ along the eigenvector $r_{0}$ and associated vector $r_{1}$, and the third derivative of $F$ along $r_{0}$. The third derivative of the flux function is very important. For example, if $d^{3} F\left(r_{0}, r_{0}, r_{0}\right)=0,(5.12)$ yields

$$
\rho_{ \pm}=\frac{1}{2}\left(l_{0} d^{2} F\left(r_{0}, r_{1}\right) \pm\left|3 l_{0} d^{2} F\left(r_{0}, r_{1}\right)-2 l_{1} d^{2} F\left(r_{0}, r_{0}\right)\right|\right) .
$$

According to (4.4),

$$
2 q \cdot r_{0}=l_{0} d^{2} F\left(r_{0}, r_{1}\right)+l_{1} d^{2} F\left(r_{0}, r_{0}\right) .
$$

Let $q \cdot r_{0}>0$, which can always be achieved by choosing the direction of $r_{0}$. Then it is straightforward to check that only the cases $b, c$, and $d$ of Table I are possible. Therefore, only these three types of singularities may appear for fluxes $F$ described by quadratic functions of state variables, see [17]. In the cases $a, e$, and $f$, the third derivative $d^{3} F\left(r_{0}, r_{0}, r_{0}\right)$ is necessarily nonzero. This result is summarized in the last column of Table I, listing the lowest degree polynomial flux functions for which each particular singularity can exist.

\subsection{Rarefaction Waves with Exceptional States}

First, let us consider the case $U \in \mathbb{R}^{2}$. Then $(\xi, \eta)$ provide the local parametrization of state space near the exceptional point $U_{*}$. Rarefaction curves of the first and second families are obtained from the integral curves in $(\xi, \eta)$ plane by folding it smoothly about the $\eta$ axis and projecting, see Figure 2. In this way, the lower part $(\xi<0)$ gives 1 -family rarefaction curves, and the upper part $(\xi>0)$ gives 2 -family rarefaction curves. The structures of rarefaction curves in state space for the six singularities of Figure 4 are presented in Figure 5. Here 1-family and 2-family rarefactions are shown by thin and bold lines respectively, and the arrows indicate the direction of increasing speed $\lambda$. Thin and bold dashed lines represent the inflection loci for 1- and 2-family rarefactions, where the corresponding speed $\lambda$ reaches an extremum (maximum or minimum can be 
a)

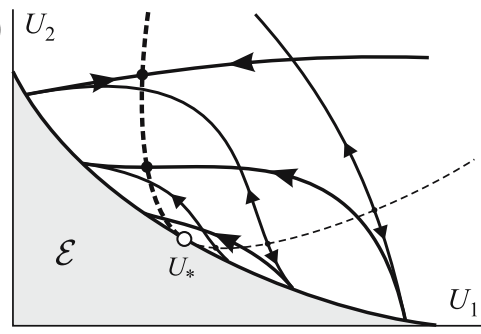

c)

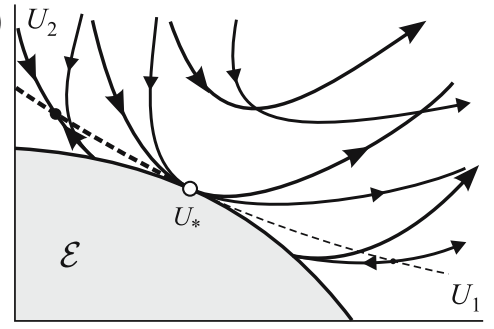

e)

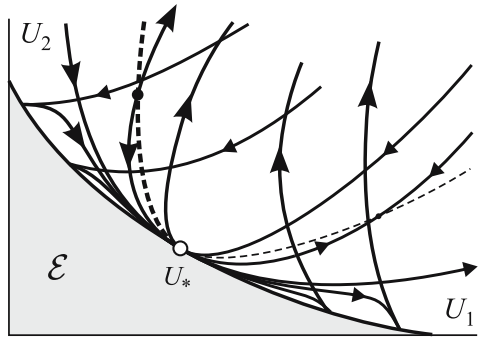

b)

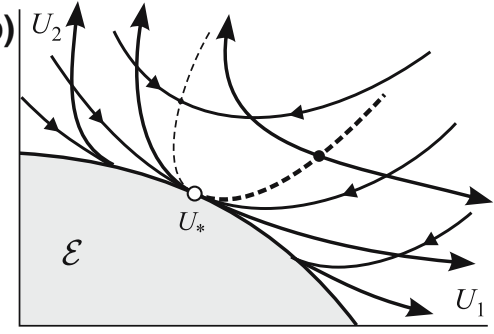

d)

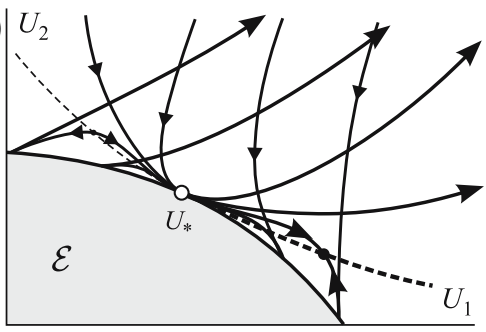

f)

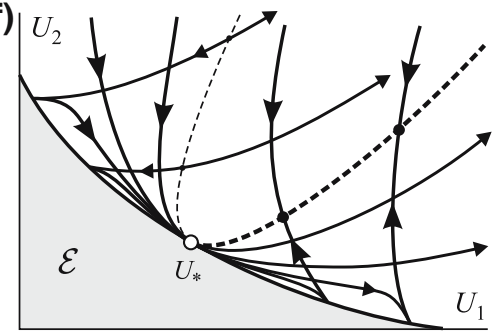

Figure 5. Generic structures of rarefaction curves near exceptional points for two conservation laws (1-family: thin curves, 2-family: bold curves, dashed inflection curves).

distinguished by means of the arrows). Notice that all the exceptional rarefactions (i.e. passing through $U_{*}$ ) as well as the inflection locus are tangent to the elliptic boundary. There can be a finite or an infinite number of exceptional rarefaction curves. In the latter case, the exceptional rarefactions span a region in state space; its boundary is determined by the integral curves tangent to the eigendirection of the larger $\left|\rho_{ \pm}\right|$.

For general $U \in \mathbb{R}^{m}$, exceptional points appear at coinciding characteristic speeds $\lambda_{i}\left(U_{*}\right)=\lambda_{i+1}\left(U_{*}\right)$ under the singularity condition $n \cdot r_{0}=0$. The integral curves in the $(m+2)$-dimensional space $(\xi, \eta, U)$ form two-dimensional invariant leaves near $\left(0,0, U_{*}\right)$ with the integral curve structure as described in Section 5.3. When rarefaction curves are reconstructed from the integral curves, these leaves projected into state space $U$ exhibit a fold 


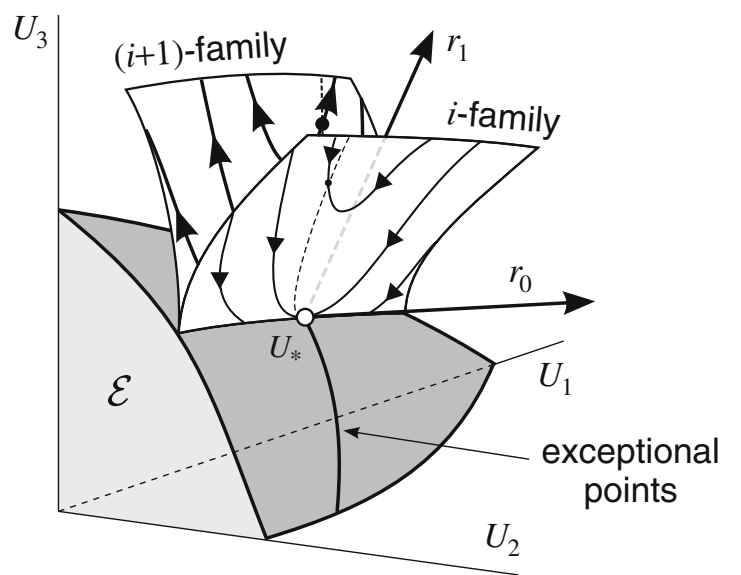

Figure 6. Rarefaction curves near exceptional point of type $b$ for $m>2$.

singularity along the $\eta$ axis, see Figure 2. Thus, two invariant two-dimensional surfaces containing rarefaction curves arise near each exceptional point in state space, see Figure 6 . One of the surfaces contains $i$-family rarefactions and the other contains $(i+1)$-family rarefactions. Since there is no relation between $i$ - and $(i+1)$-rarefactions away from the elliptic boundary, these two invariant surfaces do not coincide in general. These surfaces coincide at the elliptic boundary, which corresponds to the projection of the fold from the $(\xi, \eta, U)$ space.

The eigenvector $r$ given by (3.9) is tangent to the $i$ and $(i+1)$ invariant surfaces (when taking minus and plus signs, respectively). Using (3.7) in (3.9), we obtain

$$
r(U)=r_{0}+r_{1} \sqrt{n \cdot\left(U-U_{*}\right)}+o\left(\left\|U-U_{*}\right\|^{1 / 2}\right) .
$$

The vectors $r_{0}$ and $r_{1}$ of the two leading terms in (5.15) are tangent to the invariant surfaces. Hence, the orientation of the $i$ and $(i+1)$ invariant surfaces near the exceptional point is given by the eigenvector $r_{0}$ and the associated vector $r_{1}$, see Figure 6 .

We note that, for two conservation laws, singularities of rarefaction curves in state space have the structure of generic folded phase portraits appearing in implicit differential equations, see e.g. [1,6]. But, since our singularities have at most two nonzero eigenvalues, this analogy does not hold for three or more conservation laws.

Let us find explicitly the rarefaction curves containing the exceptional point $U_{*}$. These curves exist if $\rho_{ \pm}$are real (when the equilibrium in the 

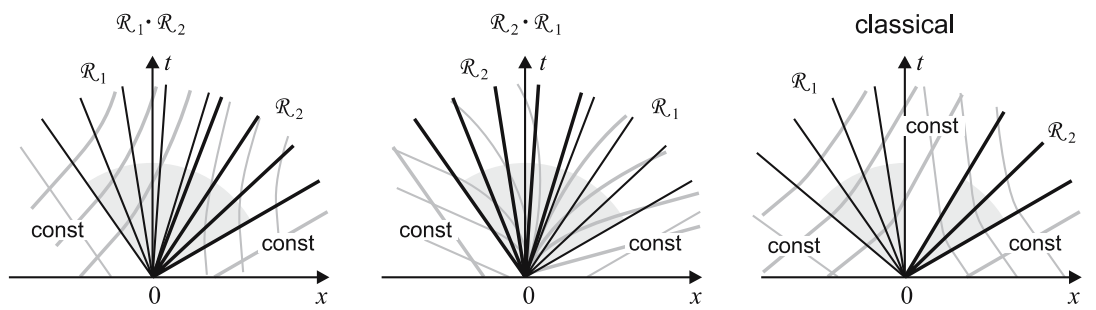

Figure 7. In the left and center figures, characteristic curves for rarefaction waves with exceptional states are compared to the classical case on the right.

lifted space is a node or saddle). Using (5.10) and solving the linearized equations (5.6), we find the integral curves starting (or finishing) at $(\xi, \eta, U)=\left(0,0, U_{*}\right)$ as

$$
\xi=\rho_{ \pm} z+o(z), \quad \eta=2\left(q \cdot r_{0}\right) z+o(z), \quad U=U_{*}+2 r_{0} z+o(z),
$$

where $z= \pm \exp \rho_{ \pm} \tau ; \rho_{+}$and $\rho_{-}$correspond to the integral curves tangent to the eigenvectors $w_{+}$and $w_{-}$, respectively. Since $\lambda=\lambda_{0}+\xi+\eta$, we find

$$
U(\lambda)=U_{*}+\frac{2\left(\lambda-\lambda_{0}\right)}{\rho_{ \pm}+2 q \cdot r_{0}} r_{0}+o\left(\lambda-\lambda_{0}\right),
$$

where $\rho_{ \pm}+2 q \cdot r_{0} \neq 0$ is the pertinent nondegeneracy condition. As opposed to the regular case (4.6), we see that the derivative of the state $U$ with respect to $\lambda$ is nonzero at the exceptional point.

The rarefaction wave solution $U(\lambda), \lambda=x / t$ can be constructed for any pair of rarefaction waves containing the state $U_{*}$, the first ending and the second starting at $U_{*}$ with increasing $\lambda$. In cases $b-f$ of Figure 5 there are two or infinite number of choices for each of these waves. We will denote such sequences of two rarefaction waves by $\mathcal{R}_{j} \cdot \mathcal{R}_{k}$, where $j, k \in$ $\{i, i+1\}$ are family numbers. Such a pair of rarefaction waves forms a single rarefaction wave in physical space. According to (5.17), the profile $U(\lambda)$ of the rarefaction wave $\mathcal{R}_{j} \cdot \mathcal{R}_{k}$ is a continuous function of $\lambda$ at $U_{*}$, which has left and right derivatives. These derivatives coincide $(U(\lambda)$ is differentiable at $\lambda=\lambda_{0}$ ) if the rarefaction curves $\mathcal{R}_{j}$ and $\mathcal{R}_{k}$ correspond to the same eigenvalue $\rho_{+}$or $\rho_{-}$. Otherwise, the derivative $d U / d \lambda$ is discontinuous at $\lambda_{0}$. Recall that the rarefaction curve $\mathcal{R}_{j}$ (as well as $\mathcal{R}_{k}$ ) is the projection of the integral curve, which is tangent to the eigendirection $w_{-}$or $w_{+}$corresponding to $\rho_{ \pm}$, see Figures 4 and 5 .

Figure 7 shows the behavior of characteristic curves in $(x, t)$ plane for the waves $\mathcal{R}_{1} \cdot \mathcal{R}_{2}$ and $\mathcal{R}_{2} \cdot \mathcal{R}_{1}$ in two conservation laws compared to the classical situation; the characteristic curves of two families are given by the equation $\mathrm{d} x / \mathrm{d} t=\lambda_{i}, i=1,2$, and are shown by thin and bold curves, 
respectively. The conserved quantities $U$ are transported along the straight black characteristic lines forming a single fan for the two families (the fan is continuous but not necessarily $\mathrm{C}^{1}$ ). Similar pictures can be drawn for the waves $\mathcal{R}_{1} \cdot \mathcal{R}_{1}$ and $\mathcal{R}_{2} \cdot \mathcal{R}_{2}$. For more than two conservation laws, interacting $i$ and $i+1$ families determine similar fans of characteristics; the other characteristics pass through these fans.

\section{RIEMANN SOLUTIONS NEAR THE ELLIPTIC BOUNDARY}

Consider the Riemann problem for the system of conservation laws (2.1), i.e., finding a weak scale-invariant solution $U(x, t)=\tilde{U}(x / t)$ for the piecewise constant initial data with a single jump at $x=0$ :

$$
U(x, 0)=\left\{\begin{array}{l}
U_{L}, x<0 \\
U_{R}, x>0
\end{array}\right.
$$

In this section, we study the Riemann problem for two conservation laws with the initial conditions $U_{L}$ and $U_{R}$ taken in the hyperbolic region near the elliptic boundary. Our main concern is to describe the nature of Riemann solutions, which contain rarefaction waves with states on the elliptic boundary. We describe a number of interesting new phenomena, rather than give a comprehensive study of Riemann solutions near the elliptic boundary.

The Riemann solution is a sequence of rarefaction and/or shock waves separated by constant states. There are two types of rarefaction waves $\mathcal{R}_{1}$ and $\mathcal{R}_{2}$, corresponding to the first and second characteristic families. Shock waves are the discontinuities consisting of a left state $U_{-}=$ $\lim _{x / t \nearrow_{s}} \tilde{U}(x / t)$ and a right state $U_{+}=\lim _{x / t \backslash s} \tilde{U}(x / t)$ propagating with shock speed $s$. The left and right states $U_{ \pm}$of the shock are related to its speed $s$ by the Rankine-Hugoniot conditions (see e.g. [26])

$$
F\left(U_{+}\right)-F\left(U_{-}\right)=s\left(U_{+}-U_{-}\right) .
$$

Let us describe classical shocks. These are 1-family shocks $\mathcal{S}_{1}$ and 2-family shocks $\mathcal{S}_{2}$, which are defined by the Lax conditions

$$
\begin{aligned}
& \mathcal{S}_{1}: \lambda_{1}\left(U_{+}\right)<s<\lambda_{1}\left(U_{-}\right), s<\lambda_{2}\left(U_{+}\right), \\
& \mathcal{S}_{2}: \lambda_{2}\left(U_{+}\right)<s<\lambda_{2}\left(U_{-}\right), s>\lambda_{1}\left(U_{-}\right),
\end{aligned}
$$

where $\lambda_{1}(U)<\lambda_{2}(U)$ are the characteristic speeds. Given a left state, say $U_{L}$, all possible right states that can be reached by a single wave are described by two curves corresponding to 1- and 2-families as shown in Figure 8 (rarefactions and shocks are represented by solid and dotted lines, respectively). If $U_{L}$ lies in the region of strict hyperbolicity, the 


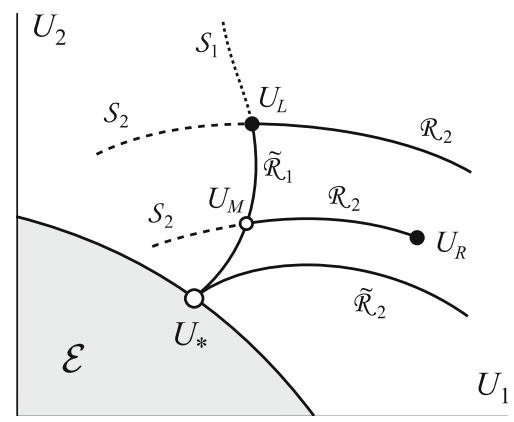

Figure 8. Riemann solutions near regular points of the elliptic boundary.

curves for the rarefaction and shock waves of the same family start at $U_{L}$ in opposite directions with the same tangent, see [26]. If $U_{L}$ belongs to the elliptic boundary, both rarefaction and shock curves have singularities, see e.g. [14].

Clearly, the speed $\lambda=x / t$ should increase from the left to the right side of the Riemann solution. According to (6.3), if two waves are separated by a constant state (represented by an arrow), they are $\mathcal{R}_{1} \rightarrow \mathcal{R}_{2}$, $\mathcal{R}_{1} \rightarrow \mathcal{S}_{2}, \mathcal{S}_{1} \rightarrow \mathcal{R}_{2}$, or $\mathcal{S}_{1} \rightarrow \mathcal{S}_{2}$. These four sequences of two waves are the only possible (classical) Riemann solutions with all states in the region of strict hyperbolicity, i.e., away from the elliptic boundary. Figure 8 shows the state space projection of the Riemann solution having type $\mathcal{R}_{1} \rightarrow \mathcal{R}_{2}$ with the initial conditions $\left(U_{L}, U_{R}\right)$ and middle constant state $U_{M}$.

In the presence of rarefaction curves that touch the elliptic boundary, new types of Riemann solutions appear. Let us start by considering a rarefaction wave containing a regular state of the elliptic boundary $U_{*}$. This wave consists of two rarefactions $\widetilde{\mathcal{R}}_{1}$ and $\widetilde{\mathcal{R}}_{2}$ (denoted by tildes) connected at $U_{*}$, see Figure 8 . Since the speed $\lambda$ changes continuously from one curve to the other, we obtain a single rarefaction wave $\widetilde{\mathcal{R}}_{1} \cdot \widetilde{\mathcal{R}}_{2}$ without a separating constant state between $\widetilde{\mathcal{R}}_{1}$ and $\widetilde{\mathcal{R}}_{2}$. In the figure, different Riemann solutions with the fixed left state $U_{L}$ are shown. One can see that the second part of the rarefaction curve $\widetilde{\mathcal{R}}_{2}$ serves as a bifurcation boundary for Riemann solutions. Indeed, if the right initial state $U_{R}$ is above $\widetilde{\mathcal{R}}_{2}$, the classical Riemann solution with the initial data $\left(U_{L}, U_{R}\right)$ exists. But the Riemann solution of this type cannot be continuously extended for the states $U_{R}$ below $\widetilde{\mathcal{R}}_{2}$. Analogously, given the right initial state $U_{R}$ lying in $\widetilde{\mathcal{R}}_{2}$, the rarefaction curve $\widetilde{\mathcal{R}}_{1}$ serves as a bifurcation boundary for Riemann solutions with different left initial states $U_{L}$.

Now let us consider Riemann solutions containing an exceptional state $U_{*}$ at the elliptic boundary. The point $U_{*}$ connects two rarefaction 

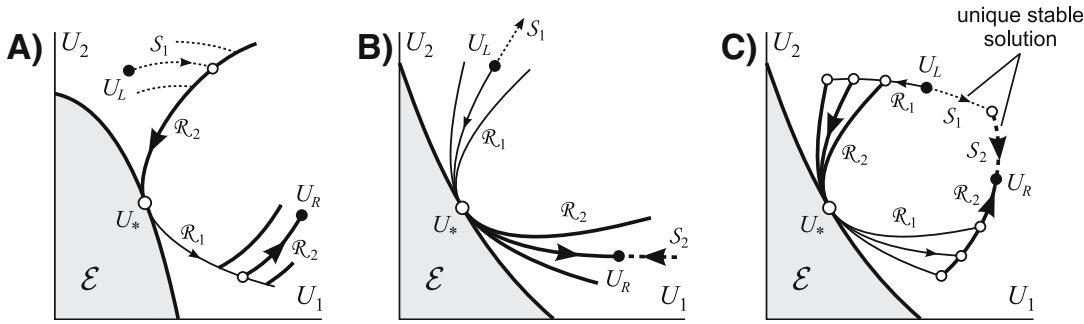

Figure 9. Nonclassical Riemann solutions in phase space near exceptional points of the elliptic boundary: (A) with transitional rarefaction (case $c$ ), (B) one-wave solution (case $d$ ), (C) infinite number of unstable solutions (cases $e$ and $f$ ).

waves as $\mathcal{R}_{1} \cdot \mathcal{R}_{1}, \mathcal{R}_{1} \cdot \mathcal{R}_{2}, \mathcal{R}_{2} \cdot \mathcal{R}_{1}$, or $\mathcal{R}_{2} \cdot \mathcal{R}_{2}$. Depending on the singularity (see Figure 5), all four possibilities can be realized.

Case $a$ : this case is remarkable: despite the existence of an eigenvector there are no rarefaction curves through the exceptional point at all, see Figure 5(a). Moreover, the Hugoniot curve through this point may reduce to the point itself.

Case $b$ : as in the regular case, the point $U_{*}$ may connect only the rarefactions $\mathcal{R}_{1}$ and $\mathcal{R}_{2}$, see Figure $5(\mathrm{~b})$. These rarefactions determine bifurcation boundaries for different types of Riemann solutions. Considering Riemann solution of type $\mathcal{R}_{1} \cdot \mathcal{R}_{2}$ that contain $U_{*}$, we see that the corresponding initial conditions form a set of zero measure in the space $\left(U_{L}, U_{R}\right)$.

Case $c$ : the point $U_{*}$ may connect two rarefactions of any types, see Figure 5(c). The interesting case is the sequence $\mathcal{R}_{2} \cdot \mathcal{R}_{1}$. This composition is known as a transitional rarefaction [10,16] (the example in [16] corresponds to case VI of Schaeffer-Shearer classification [21] for planar quadratic polynomial fluxes). Transitional rarefactions appear in Riemann solutions between the first and second family waves, e.g. $\mathcal{S}_{1} \rightarrow \mathcal{R}_{2} \cdot \mathcal{R}_{1} \rightarrow$ $R_{2}$, see Figure 9(A) (the arrows in the figure indicate increasing speed $\lambda=$ $x / t$ ). Analyzing the figure, we find the following properties of Riemann solutions. If, for some initial condition $\left(U_{L}, U_{R}\right)$, there is a Riemann solution containing a transitional rarefaction, then similar Riemann solutions exist for any initial condition in some neighborhood of $\left(U_{L}, U_{R}\right)$. Moreover, such Riemann solutions are unique (at least locally). Therefore, transitional rarefactions appear in Riemann solutions in a structurally stable way.

The remaining three cases $d$, $e$, and $f$ provide new types of nonclassical Riemann solutions. 
Case $d$ : the point $U_{*}$ connects only rarefactions of types $\mathcal{R}_{1}$ and $\mathcal{R}_{2}$. But, unlike case $b$, there are infinite numbers of $\mathcal{R}_{1}$ and $\mathcal{R}_{2}$ rarefactions, see Figure 5(d). Riemann solutions in this case have no extra waves, i.e., their structure is $\mathcal{R}_{1} \cdot \mathcal{R}_{2}$, see Figure $9(\mathrm{~B})$. Since there are one-parameter families of rarefaction curves for both $\mathcal{R}_{1}$ and $\mathcal{R}_{2}$, such Riemann solutions are structurally stable, i.e., they are unique and persist under arbitrary small change of the initial data. We remark that the structure of shock curves $\mathcal{S}_{1}$ and $\mathcal{S}_{2}$ at the points $U_{L}$ and $U_{R}$ does not allow local Riemann solutions containing shock waves, see Figure 9(B).

Cases $e$ and $f$ : these two cases are similar. The point $U_{*}$ connects rarefactions $\mathcal{R}_{2}$ and $\mathcal{R}_{1}$. Each of them can be chosen from the corresponding one-parameter family of rarefaction curves, see Figure 5(e, f). There can be two extra waves at the beginning and at the end of the Riemann solution, e.g. $\mathcal{R}_{1} \rightarrow \mathcal{R}_{2} \cdot \mathcal{R}_{1} \rightarrow R_{2}$, see Figure $9(\mathrm{C})$. At first sight, the situation is similar to the transitional wave case $c$ in Figure 9(A). However, there is an infinite number of possible choices for the middle wave, so that there exist an infinite number of Riemann solutions (more precisely, a two-parameter family of Riemann solutions) for the same initial conditions $\left(U_{L}, U_{R}\right)$. Moreover, under an arbitrarily small perturbation of the initial Riemann data, this infinite number of solutions persists. Definitely, this situation is completely unnatural from the physical point of view.

To resolve this paradox, consider a solution for the system of conservation laws (2.1) with continuous initial conditions that change from $U_{L}$ to $U_{R}$ in a small space interval $-\delta \leq x \leq \delta$ with the states lying inside the region of strict hyperbolicity. For example,

$$
U(x, 0)=\left\{\begin{array}{lc}
U_{L}, & x<-\delta ; \\
\left(U_{R}-U_{L}\right) x /(2 \delta)+\left(U_{R}+U_{L}\right) / 2, & -\delta \leq x \leq \delta \\
U_{R}, & x>\delta .
\end{array}\right.
$$

At large times $t$, this solution should asymptotically approach the Riemann solution with the initial conditions (6.1). Out of the infinite number of Riemann solutions described above, which would be the limit? The answer is: none of them. The limit Riemann solution is classical and consists of two shocks $\mathcal{S}_{1} \rightarrow \mathcal{S}_{2}$ as shown in Figure $9(\mathrm{C})$. Our hypothesis is supported by a number of numerical experiments that we have done for different systems of two conservation laws with small viscosity. We note that similar numerical experiments showed stability of Riemann solutions for the cases in Figure 9(A,B). 


\section{EXAMPLE}

As an example, consider a system of two conservation laws with the quadratic flux function

$$
F(U)=\left(\begin{array}{c}
U_{2}+\alpha U_{1}^{2} / 2+U_{2}^{2} / 2 \\
10 U_{1} U_{2}
\end{array}\right), \quad U=\left(\begin{array}{c}
U_{1} \\
U_{2}
\end{array}\right),
$$

where $\alpha$ is a real parameter. Evaluating characteristic speeds of the system, we find the elliptic region $\mathcal{E}$ as

$$
40 U_{2}\left(1+U_{2}\right)+(\alpha-10)^{2} U_{1}^{2}<0,
$$

which is an ellipse in state space. Let us consider a point $U_{*}=0$ at the elliptic boundary. At this point, the double characteristic speed is $\lambda_{0}=0$ with the right and left eigenvectors and associated vectors

$$
r_{0}=\left(\begin{array}{l}
1 \\
0
\end{array}\right), \quad r_{1}=\left(\begin{array}{l}
0 \\
1
\end{array}\right), \quad l_{0}=\left(\begin{array}{l}
0 \\
1
\end{array}\right), \quad l_{1}=\left(\begin{array}{l}
1 \\
0
\end{array}\right),
$$

satisfying the normalization conditions (3.4). Using formula (3.11), we find $n=(0,10)$. Hence $n \cdot r_{0}=0$, i.e., the eigenvector $r_{0}$ is tangent to the elliptic boundary and $U_{*}$ is the exceptional point. Substituting (7.1), (7.3) into expressions (5.13) and (5.14), we obtain

$$
\rho_{ \pm}=(10 \pm|30-2 \alpha|) / 2, \quad 2 q \cdot r_{0}=\alpha+10 .
$$

Here we assume that $\alpha+10>0$ (recall that $q \cdot r_{0}>0$ was required for the classification in Table I). For $\alpha+10<0$ we take opposite signs for all the vectors in (7.3). Then $2 q \cdot r_{0}=-(\alpha+10)>0$ and $\rho_{ \pm}=(-10 \pm \mid 30-$ $2 \alpha \mid) / 2$. According to the Table I, three types of singularities $(b, c$, and $d$ in Figures 4,5 ) are possible depending on $\alpha$ :

$$
b: \alpha<0 ; \quad c: 0<\alpha<10 \text { or } 20<\alpha ; \quad d: 10<\alpha<20 .
$$

Consider the particular case when $\alpha=14$ (case $d$ ). The structure of exceptional rarefaction curves for this case is shown in Figure 10(a) (remark that the two lower curves pass through two other exceptional points that correspond to case $c$ ). There is an infinite number of $\mathcal{R}_{1} \cdot \mathcal{R}_{2}$ rarefaction curves passing through the exceptional point $U_{*}$. More precisely, the $\mathcal{R}_{1} \cdot \mathcal{R}_{2}$ rarefaction curves form a two-parameter family consisting of a one-parameter family of $R_{1}$ curves and a one-parameter family of $R_{2}$ curves. Thus, single rarefaction wave Riemann solutions are found for any initial conditions such that $U_{L}$ and $U_{R}$ belong to the regions spanned by the $R_{1}$ and $R_{2}$ exceptional rarefaction curves, respectively. 
a)

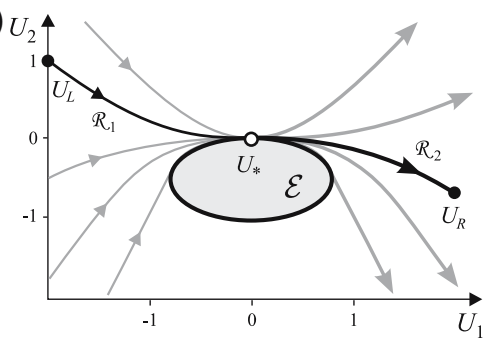

b)

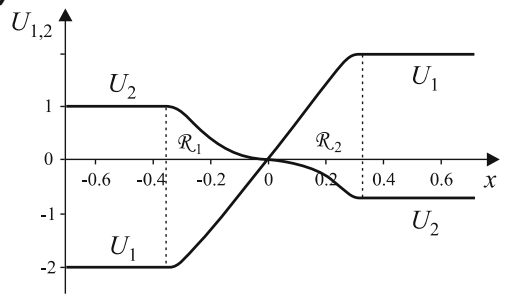

Figure 10. Singular rarefaction wave $\mathcal{R}_{1} \cdot \mathcal{R}_{2}$ for two specific conservation laws: (a) in state space (b) in numerical Riemann solution.

Numerical experiments were carried out using a linearized CrankNicolson scheme for two conservation laws (2.1), (7.1) (with the additional viscous term $0.01 \partial^{2} U / \partial x^{2}$ ). As the initial conditions we took (6.4) with $U_{L}=(-2,1), U_{R}=(2,-0.7)$, and $\delta=0.01$. Figure 10 (b) shows the numerical solution at time $t=0.01$. This solution represents a single rarefaction wave of type $\mathcal{R}_{1} \cdot \mathcal{R}_{2}$. The profile $U(\lambda), \lambda=x / t$ of this wave is differentiable at $\lambda_{0}=0$, since both $\mathcal{R}_{1}$ and $\mathcal{R}_{2}$ correspond to the same eigenvalue $\rho_{-}=4$, see (5.17). The functions $U_{1}(x, t)$ and $U_{2}(x, t)$ intersect the $x$-axis at the same point, which corresponds to the exceptional state $U_{*}=0$. By taking initial conditions $U(x, 0)$ different from (6.4), we observed solutions with the same asymptotic behavior. Thus, the Riemann solution consisting of a single rarefaction wave passing through the exceptional point is stable in the Hadamard sense. Similar solutions were obtained for perturbed initial data $\left(U_{L}, U_{R}\right)$ showing structural stability of our one-wave solution. Numerical analysis of the Hugoniot and rarefaction curves showed that the resulting Riemann solution is the unique solution for the given initial data.

We note that if the linear term is removed from (7.1), the elliptic region shrinks to an umbilic point, and the flux corresponds to case III of Schaeffer-Shearer classification [21]. The double-family rarefaction wave solutions persist in this limit, see [20], where analogous solutions passing through the umbilic point were described.

\section{CONCLUSION}

In this paper, we studied singularities of rarefaction waves in systems of conservation laws near the boundary of the elliptic region. Regular and exceptional points at the elliptic boundary are distinguished, and the local structure of rarefaction curves is studied in detail. We emphasize that the 
theory provides not only qualitative, but also quantitative results that can be used for finding the type of singularity and structure of nearby rarefaction curves. The necessary information includes eigenvectors, associated vectors, and derivatives up to third order of the flux function evaluated at the singularity. We note the importance of the third derivatives of the flux function: using only quadratic local approximations may lead to incorrect singularity type and rarefaction structure.

Singularities of rarefaction waves at exceptional points are related to nonclassical Riemann solutions, which are stable both structurally and in the Hadamard sense. A new type of Riemann solution was detected in this way.

A surprise, detected for some types of singularities, was the existence of an infinite number of structurally stable Riemann solutions for the same initial conditions. This paradox was resolved by numerical simulations, which showed that all these Riemann solutions, except for the classical one, are unstable in the Hadamard sense.

In spirit, our work is similar to the study [22] of full wave curves for two conservation laws. In that paper, elliptic regions were ruled out; here we provide the theory for singularities of rarefaction waves needed to extend that work. We note that in the generic situation considered in our paper, the hyperbolic region is not invariant under the flow of the evolution partial differential equation (2.1), as discussed in [18]. The latter work also describes models with unbounded elliptic regions with no exceptional boundary points. However, these aspects fall out of the scope of the current work.

Of course, the study of shock waves and their admissibility in the presence of elliptic regions is also necessary. Surprising phenomena may be associated with singularities of Hugoniot curves near exceptional points. Singularities at the elliptic boundaries associated with the coincidence of three or more characteristic speeds are also of interest [19].

\section{APPENDIX A}

Separating the first and second columns in equation (3.6), we obtain

$$
\begin{aligned}
& A(U) R_{0}(U)=\left(\lambda_{0}+s(U)\right) R_{0}(U)+p(U) R_{1}(U), \\
& A(U) R_{1}(U)=\left(\lambda_{0}+s(U)\right) R_{1}(U)+R_{0}(U) .
\end{aligned}
$$

At the eigenvalue coupling point $U_{*}$, we have $s\left(U_{*}\right)=p\left(U_{*}\right)=0$. Then equations (A.1) and (A.2) coincide with (3.1), i.e., $R_{0}\left(U_{*}\right)=r_{0}$ and $R_{1}\left(U_{*}\right)=r_{1}$. Taking the derivative of both sides of (A.1) and (A.2) with respect to state variable at $U_{*}$, we get 


$$
\begin{aligned}
& \left(A_{0}-\lambda_{0} I\right) \frac{\partial R_{0}}{\partial U_{k}}=\frac{\partial s}{\partial U_{k}} r_{0}+\frac{\partial p}{\partial U_{k}} r_{1}-\frac{\partial A}{\partial U_{k}} r_{0}, \\
& \left(A_{0}-\lambda_{0} I\right) \frac{\partial R_{1}}{\partial U_{k}}=\frac{\partial R_{0}}{\partial U_{k}}+\frac{\partial s}{\partial U_{k}} r_{1}-\frac{\partial A}{\partial U_{k}} r_{1} .
\end{aligned}
$$

Pre-multiplying equation (A.3) by the left eigenvector $l_{0}$ and using relations (3.2)-(3.4), we obtain

$$
\frac{\partial p}{\partial U_{k}}=l_{0} \frac{\partial A}{\partial U_{k}} r_{0} .
$$

Similarly, by taking the sum of equations (A.3) and (A.4) pre-multiplied by $l_{1}$ and $l_{0}$, respectively, we find

$$
\frac{\partial s}{\partial U_{k}}=\frac{1}{2}\left(l_{1} \frac{\partial A}{\partial U_{k}} r_{0}+l_{0} \frac{\partial A}{\partial U_{k}} r_{1}\right) .
$$

Let us introduce the nonsingular matrix

$$
G=A_{0}-\lambda_{0} I+r_{1} l_{1}
$$

If, for given $b$, the singular linear equation $\left(A_{0}-\lambda_{0} I\right) x=b$ has a solution, then $x=G^{-1} b$ is the unique solution satisfying the condition $l_{1} x=0$, see [24]. Using (3.1)-(3.4), one can easily check the relations

$$
G r_{0}=r_{1}, \quad G r_{1}=r_{0}, \quad l_{0} G=l_{1}, \quad l_{1} G=l_{0} .
$$

Now, having the right-hand sides of equations (A.3) and (A.4), we find their particular solutions by using the matrix $G$ as

$$
\begin{aligned}
& \frac{\partial R_{0}}{\partial U_{k}}=G^{-1}\left(\frac{\partial s}{\partial U_{k}} r_{0}+\frac{\partial p}{\partial U_{k}} r_{1}-\frac{\partial A}{\partial U_{k}} r_{0}\right)=\frac{\partial s}{\partial U_{k}} r_{1}+\frac{\partial p}{\partial U_{k}} r_{0}-G^{-1} \frac{\partial A}{\partial U_{k}} r_{0}, \\
& \frac{\partial R_{1}}{\partial U_{k}}=G^{-1}\left(\frac{\partial R_{0}}{\partial U_{k}}+\frac{\partial s}{\partial U_{k}} r_{1}-\frac{\partial A}{\partial U_{k}} r_{1}\right)=\frac{\partial s}{\partial U_{k}} r_{0}+G^{-1}\left(\frac{\partial R_{0}}{\partial U_{k}}-\frac{\partial A}{\partial U_{k}} r_{1}\right) .
\end{aligned}
$$

We remark that the general solutions are obtained by adding an arbitrary multiple of the eigenvector $r_{0}$ to the right-hand sides of (A.9) and (A.10). According to the versal deformation theory $[12,13]$, any choice for the solution is correct.

Finally, we find the second derivative $d^{2} p\left(r_{0}, r_{0}\right)$ under the condition $n \cdot r_{0}=0$. For this purpose, we take the second derivative along $r_{0}$ of both sides of (A.1) and obtain

$$
\begin{aligned}
\left(A_{0}-\lambda_{0} I\right) d^{2} R_{0}\left(r_{0}, r_{0}\right)= & r_{0} d^{2} s\left(r_{0}, r_{0}\right)+2\left(q \cdot r_{0}\right) d R_{0}\left(r_{0}\right)+r_{1} d^{2} p\left(r_{0}, r_{0}\right) \\
& -2 d A\left(r_{0}\right) d R_{0}\left(r_{0}\right)-d^{2} A\left(r_{0}, r_{0}\right) r_{0} .
\end{aligned}
$$


Pre-multiplying (A.11) by $l_{0}$ and using (3.2)-(3.4), (A.6), (A.8), (A.9), yields

$$
d^{2} p\left(r_{0}, r_{0}\right)=2\left(q \cdot r_{0}\right)^{2}-2 l_{0} d A\left(r_{0}\right) G^{-1} d A\left(r_{0}\right) r_{0}+l_{0} d^{2} A\left(r_{0}, r_{0}\right) r_{0}
$$

Similarly, if $n \cdot r_{0}=0$ we obtain

$$
\begin{aligned}
d^{2} p\left(a, r_{0}\right)= & 2\left(q \cdot r_{0}\right)(q \cdot a)-l_{0} d A\left(r_{0}\right) G^{-1} d A(a) r_{0}-l_{0} d A(a) G^{-1} d A\left(r_{0}\right) r_{0} \\
& +(n \cdot a) l_{1} d A\left(r_{0}\right) r_{1}+l_{0} d^{2} A\left(a, r_{0}\right) r_{0}, \quad a \in \mathbb{R}^{m} .
\end{aligned}
$$

\section{ACKNOWLEDGMENTS}

This work was supported by $\mathrm{CNP}_{\mathrm{q}}$ under Grants 301532/2003-6 and 300097/2004-2, FAPERJ under Grant E-26/152.163/2002, CAPES under Grant 0722/2003 (PAEP no. 0143/03-00), and President of RF grant No. MK-2012.2006.1.

\section{REFERENCES}

1. Anosov, D. V., and Arnold, V. I., eds. (1988). Dynamical Systems. I. Ordinary Differential Equations and Smooth Dynamical Systems, Springer, Berlin.

2. Arnold, V. I. (1983). Geometrical Methods in the Theory of Ordinary Differential Equations, Springer, New York.

3. Azevedo, A. V., and Marchesin, D. (1990). Multiple viscous profile Riemann solutions in mixed elliptic-hyperbolic models for flow in porous media. In Nonlinear Evolution Equations that Change Type, IMA Vol. Math. Appl. 27, Springer, New York.

4. Azevedo, A. V., Marchesin, D., Plohr, B., and Zumbrun, K. (2002). Capillary instability in models for three-phase flow, Angew, Z.,. Math. Phys. 53, 713-746.

5. Bell, J. B., Trangenstein, J. A., and Shubin, G. R. (1986). Conservation laws of mixed type describing three-phase flow in porous media. SIAM J. Appl. Math. 46, 1000-1017.

6. Dara, L. (1975). Singularités génériques des équations différentielles multiformes. Bol. Soc. Brasil. Mat. 6, 95-128.

7. Eschenazi, C. (1995). Rarefaction fields in systems of three conservation laws. Mat. Contemp. 8, 151-176.

8. Eschenazi, C., and Palmeira, C. F. B. (1998). Local topology of elementary waves for systems of two conservation laws, Mat. Contemp. 15, 127-144.

9. Isaacson, E. L., Marchesin, D., Palmeira C. F., and Plohr, B. J. (1992). A global formalism for nonlinear waves in conservation laws, Comm. Math. Phys. 146, 505-552.

10. Isaacson, E. L., Marchesin, D., and Plohr, B. J. (1990). Transitional waves for conservation laws, SIAM J. Math. Anal. 21, 837-866.

11. Keyfitz, B. L. (1995). A geometric theory of conservation laws which change type, Z. Angew. Math. Mech. 75, 571-581.

12. Mailybaev, A. A. (2000). Transformation of families of matrices to normal forms and its application to stability theory, SIAM J. Matrix Anal. Appl. 21, 396-417.

13. Mailybaev, A. A. (2001). Transformation to versal deformations of matrices, Linear Algebra Appl. 337, 87-108. 
14. Marchesin, D., and Palmeira, C. F. B. (1994). Topology of elementary waves for mixedtype systems of conservation laws, J. Dynam. Differ. Eq. 6, 427-446.

15. Marchesin, D., and Plohr, B. (2001). Wave structure in WAG recovery. Soc. Petroleum Eng. $J, 6,209-219$.

16. Matos, V. M. M. (2004). Riemann Problem for Two Conservation Laws of Type IV with Elliptic Region, Ph.D. Thesis, IMPA, Riode Janeiro (in Portuguese).

17. Palmeira, C. F. B. (1988). Line fields defined by eigenspaces of derivatives of maps from the plane to itself, Proceedings of the Sixth International Colloquium on Differential Geometry (Santiago de Compostela), pp 177-205.

18. Pego, L. R., and Serre, D. (1988). Instabilities in Glimm's scheme for two systems of mixed type, SIAM J. Numer. Anal. 25, 965-988.

19. Radicchi, R. (2005). On implicit ordinary differential equations in three dimensions, Ph.D Thesis, Universidade Federal de Minas Gerais, Minas Gerais (Brazil).

20. Schaeffer, D. G., and Shearer, M. (1987). The classification of $2 \times 2$ systems of nonstrictly hyperbolic conservation laws, with application to oil recovery, Comm. Pure Appl. Math. 40, 141-178.

21. Schaeffer, D. G., and Shearer, M. (1987). Riemann problems for nonstrictly hyperbolic $2 \times$ 2 systems of conservation laws. Trans. Amer. Math. Soc. 304, 267-306.

22. Schecter, S., Plohr, B. J., and Marchesin, D. (2001). Classification of codimension-one Riemann solutions, J. Dynam. Differ. Eq. 13, 523-588.

23. Schulte, W., and Falls, A. (1992). Features of three-component, three-phase displacement in porous media. SPE Reservoir Eng. 7, 426-432.

24. Seyranian, A. P., and Mailybaev, A. A. (2003). Multiparameter Stability Theory with Mechanical Applications, World Scientific, Singapore.

25. Shearer, M., and Trangenstein, J. (1989). Loss of Real Characteristics for Models of Three-Phase Flow in Porous Media, Transport in Porous Media 4, 499-525.

26. Smoller, J. (1983). Shock Waves and Reaction-Diffusion Equations, Springer, New York,

27. Tang, Z. J., and Ting, T. C. T. (1987). Wave curves for the Riemann problem of plane waves in isotropic elastic solids, Internat. J. Engrg. Sci. 25, 1343-1381.

28. Xin, Z. P. (1989). Asymptotic stability of rarefaction waves for $2 \times 2$ viscous hyperbolic conservation laws - the two-modes case, J. Differ. Eq. 78, 191-219. 